\title{
Error estimates for finite volume element methods for convection-diffusion-reaction equations
}

\author{
Rajen K. Sinha ${ }^{\mathrm{a}, *}$, Jürgen Geiser ${ }^{\mathrm{b}}$ \\ ${ }^{a}$ Department of Mathematics, Indian Institute of Technology Guwahati, Guwahati 781039, India \\ b Weierstrass Institute for Applied Analysis and Stochastics, Mohrenstrasse 39, D-10117 Berlin, Germany \\ Available online 19 January 2006
}

\begin{abstract}
In this paper, we study finite volume element (FVE) method for convection-diffusion-reaction equations in a two-dimensional convex polygonal domain. These types of equations arise in the modeling of a waste scenario of a radioactive contaminant transport and reaction in flowing groundwater. Both spatially discrete scheme and discrete-in-time scheme are analyzed in this paper. For the spatially discrete scheme, optimal order error estimates in $L^{2}$ and $H^{1}$ norms are obtained for the homogeneous equation using energy method. Further, a quasi-optimal order error estimate in $L^{\infty}$ norm is shown to hold in an interior subdomain away from the corners. Based on backward Euler method, a time discretization scheme is discussed and related error estimates are derived. (C) 2005 IMACS. Published by Elsevier B.V. All rights reserved.
\end{abstract}

MSC: 65M60; 65N30; 65N 15

Keywords: Convection-diffusion-reaction equation; Finite volume element method; Spatially discrete scheme; Discrete-in-time scheme; Error estimates

\section{Introduction}

Our mathematical formulation is based on a potential waste scenario of radioactive contaminants, which are transported and reacted with flowing groundwater in porous media (cf. [11-13]). The model is described in the formulation as an initial-boundary value problem of the form

$$
u_{t}+\nabla \cdot(\mathbf{v} u-\mathcal{D}(x) \nabla u)+\lambda u=f(x, t) \quad \text { in } \Omega \times J
$$

subject to the boundary conditions

$$
u=g_{1}(x, t) \quad \text { on } \Gamma_{1} ; \quad(\mathbf{v} u-\mathcal{D} \nabla u) \cdot \mathbf{n}=g_{2}(x, t) \quad \text { on } \Gamma_{2}
$$

and initial condition

$$
u(x, 0)=u_{0}(x) \text { in } \Omega .
$$

\footnotetext{
* Corresponding author.

E-mail addresses: rajen@iitg.ernet.in (R.K. Sinha), geiser@wias-berlin.de (J. Geiser).
} 
Here, $\Omega \subset \mathbb{R}^{2}$ is a bounded convex polygonal domain with boundary $\Gamma=\Gamma_{1} \cup \Gamma_{2}, J=(0, T]$ with $T<\infty$ and $u_{t}=\partial u / \partial t$. Further, $\mathcal{D}=\left\{d_{i, j}(x)\right\}$ is a symmetric and uniformly positive definite dispersion-diffusion matrix of size $2 \times 2$ in $\Omega$. The parameter $\mathbf{v}$ is the divergence free groundwater velocity and $\lambda$ is the constant reaction parameter. The nonhomogeneous term $f$ and the coefficients $d_{i j}(x)$ are assumed to be smooth for our purpose.

With the substitution $\tilde{u}=u-g_{1}$ on $\Gamma_{1}$, we rewrite Eqs. (1.1)-(1.3) as

$$
\tilde{u}_{t}+\nabla \cdot(\mathbf{v} \tilde{u}-\mathcal{D}(x) \nabla \tilde{u})+\lambda \tilde{u}=f(x, t) \quad \text { in } \Omega \times J
$$

subject to the boundary conditions

$$
\tilde{u}=0 \quad \text { on } \Gamma_{1} ; \quad(\mathbf{v} \tilde{u}-\mathcal{D} \nabla \tilde{u}) \cdot \mathbf{n}=g_{2} \quad \text { on } \Gamma_{2}
$$

and initial condition

$$
\tilde{u}(x, 0)=u_{0}(x) \text { in } \Omega \text {. }
$$

Thus, study of problem (1.1)-(1.3) now reduces to the study of equivalent problem (1.4)-(1.6).

In the recent years, the use of finite volume element methods has become popular due to its certain conservation feature that are desirable in many applications (cf. [8-10]). The FVE method considered in these paper are based on Petrov-Galerkin formulation in which solution space consisting of continuous piecewise polynomial functions and the test space consisting of piecewise constant functions. The test space essentially conserve the local conservation property of the method. In [8,9], the authors have studied this type of problem with self-adjoint elliptic operator and proved optimal $L^{2}$ and $H^{1}$ error estimates which requires higher regularity requirement on the solution when compared to that of finite element method (cf. [17,20]). Recently, the authors of [6] have studied FVE for self-adjoint parabolic problem with homogeneous Dirichlet boundary condition and derived optimal error estimate in $L^{2}$ and $H^{1}$ norms, and suboptimal order of error estimate in $L^{\infty}$ norm. They have used semigroup theory in a crucial way in their analysis.

In this present paper, we study the convergence of FVE methods for a non-selfadjoint parabolic problem. Both spatially discrete scheme and discrete-in-time scheme are discussed and optimal error estimates in $L^{2}$ and $H^{1}$ norms are proved using only energy method. In addition, a quasi-optimal order in $L^{\infty}$ norm is obtained in an interior subdomain away from the corners. Our analysis avoid the use of semigroup theory and the regularity requirement on the solution is same as that of finite element method. Further, based on backward Euler method the fully discrete scheme is analyzed and related optimal error estimates are established. To the best our knowledge error estimates for the problem (1.1)-(1.3) using FVE method have not been established earlier.

The literature on the theoretical framework and the basic tools for the analysis of the finite volume element methods for elliptic and parabolic problems are described in [3-5,7,10,15,16,18,19] and references therein.

A brief outline of this paper is as follows. In Section 2, we introduce some notations and present some preliminary materials to be used in our subsequent sections. The Petrov-Ritz projection is introduced and related estimates are carried out in Section 3. Section 4 is devoted to the error estimates for the FVE method. Finally, the backward Euler time discretization scheme is discussed in Section 5.

Throughout this paper, $C$ denotes a generic positive constant which does not depend on the spatial and time discretization parameters $h$ and $k$, respectively.

\section{Notations and preliminaries}

Let $V=\left\{\phi \in H^{1}(\Omega) \mid \phi=0\right.$ on $\left.\Gamma_{1}\right\}$. For the purpose of finite volume element approximation of (1.4)-(1.6), the weak formulation of the problem may be stated as follows: Find $\tilde{u}: \bar{J} \rightarrow V$ such that

$$
\left(\tilde{u}_{t}, \phi\right)+A(\tilde{u}, \phi)=\left\langle g_{2}, \phi\right\rangle+(f, \phi), \quad \forall \phi \in V
$$

with $\tilde{u}(0)=u_{0}$, where the bilinear form $A(\cdot, \cdot)$ is given by

$$
A(\tilde{u}, \phi)=\int_{\Omega}(\mathcal{D}(x) \nabla \tilde{u} \cdot \nabla \phi-\mathbf{v} \tilde{u} \nabla \phi+\lambda \tilde{u} \phi) \mathrm{d} x .
$$


Here and below, we denote $(\cdot, \cdot)$ and $\|\cdot\|$ by $L^{2}$ inner product and the induced norm on $L^{2}(\Omega)$. The notation $\langle\cdot, \cdot\rangle$ is used to denote boundary integral over $\Gamma_{2}$. Further, we shall use the standard notation for Sobolev spaces $W^{m, p}(\Omega)$ with $1 \leqslant p \leqslant \infty$. The norm on $W^{m, p}(\Omega)$ is defined by

$$
\|u\|_{m, p, \Omega}=\|u\|_{m, p}=\left(\int_{\Omega} \sum_{|\alpha| \leqslant m}\left|D^{\alpha} u\right|^{p} \mathrm{~d} x\right)^{1 / p}, \quad 1 \leqslant p<\infty
$$

with the standard modification for $p=\infty$. When $p=2$, we write $W^{m, 2}(\Omega)$ by $H^{m}(\Omega)$ and denote the norm by $\|\cdot\|_{m}$. For a fractional number $s$, Sobolev space $H^{s}$ is defined in [1].

Note that the bilinear form $A(\cdot, \cdot)$ given by (2.2) may not be coercive but it can be made coercive by adding a sufficiently large constant $\kappa \in \mathbb{R}$ times the $L^{2}$-inner product. That is, it satisfies Gärding's type inequality (cf. [2])

$$
A(\phi, \phi)+\kappa\|\phi\|^{2} \geqslant \frac{\alpha}{2}\|\phi\|_{1}^{2}, \quad \forall \phi \in V .
$$

Introducing the transformation $\bar{u}=e^{-\kappa t} \tilde{u}$ as a new dependent variable, we rewrite (1.4) as

$$
\bar{u}_{t}+A_{\kappa} \bar{u}=\bar{f}=e^{-\kappa t} f, \quad t \in J
$$

with $\bar{u}(0)=u_{0}$, where

$$
A_{k} \bar{u}=\nabla \cdot(\mathbf{v} \bar{u}-\mathcal{D}(x) \nabla \bar{u})+(\lambda+\kappa) \bar{u} .
$$

The weak form corresponding to (2.3) is defined to be the function $\bar{u}: \bar{J} \rightarrow V$ such that

$$
\left(\bar{u}_{t}, \phi\right)+A_{\kappa}(\bar{u}, \phi)=\left\langle\bar{g}_{2}, \phi\right\rangle+(\bar{f}, \phi), \quad \forall \phi \in V
$$

with $\bar{g}_{2}=e^{-\kappa t} g_{2}$ and $\bar{u}(0)=u_{0}$. The bilinear form $A_{\kappa}(\cdot, \cdot)$ is given by

$$
A_{\kappa}(\bar{u}, \phi)=\int_{\Omega} \mathcal{D}(x) \nabla \bar{u} \cdot \nabla \phi \mathrm{d} x-\int_{\Omega} \mathbf{v} \bar{u} \nabla \phi \mathrm{d} x+\int_{\Omega}(\lambda+\kappa) \bar{u} \phi \mathrm{d} x .
$$

\subsection{A priori estimates}

Following the lines of proof in [17], it is easy to derive a priori bounds for the solution $\bar{u}$ satisfying (2.3) under appropriate regularity assumption on the initial function $u_{0}$. The details are thus omitted.

Lemma 2.1. Let $u_{0} \in L^{2}(\Omega)$ and $g_{2} \in H^{1 / 2}\left(\Gamma_{2}\right)$. Then, for $f=0$, we have

$$
\|\bar{u}(t)\|^{2}+\int_{0}^{t}\|\bar{u}(s)\|_{1}^{2} \mathrm{~d} s \leqslant C\left(\left\|u_{0}\right\|^{2}+\int_{0}^{t}\left\|g_{2}\right\|_{H^{1 / 2}\left(\Gamma_{2}\right)}^{2} \mathrm{~d} s\right) .
$$

Moreover, when $u_{0} \in V$, we have

$$
\|\bar{u}(t)\|_{1}^{2}+\int_{0}^{t}\left\{\left\|\bar{u}_{s}(s)\right\|^{2}+\|\bar{u}(s)\|_{2}^{2}\right\} \mathrm{d} s \leqslant C\left(\left\|u_{0}\right\|_{1}^{2}+\int_{0}^{t}\left\|g_{2}\right\|_{H^{1 / 2}\left(\Gamma_{2}\right)}^{2} \mathrm{~d} s\right) .
$$

Lemma 2.2. Assume that $u_{0} \in H^{2}(\Omega) \cap V, \frac{\partial^{j} g_{2}}{\partial t^{j}} \in H^{1 / 2}\left(\Gamma_{2}\right)(j=0,1,2)$ and $f=0$. Then, we have

$$
\begin{aligned}
& \left\|\bar{u}_{t}(t)\right\|^{2}+\int_{0}^{t}\left\|\bar{u}_{s}(s)\right\|_{1}^{2} \mathrm{~d} s \leqslant C\left(\left\|u_{0}\right\|_{2}^{2}+\sum_{j=0}^{1} \int_{0}^{t}\left\|\frac{\partial^{j} g_{2}}{\partial t^{j}}\right\|_{H^{1 / 2}\left(\Gamma_{2}\right)}^{2} \mathrm{~d} s\right), \\
& t\left\|\bar{u}_{t}(t)\right\|_{1}^{2}+\int_{0}^{t} s\left\|\bar{u}_{s s}\right\|^{2} \mathrm{~d} s \leqslant C\left(\left\|u_{0}\right\|_{2}^{2}+\sum_{j=0}^{1} \int_{0}^{t}\left\|\frac{\partial^{j} g_{2}}{\partial t^{j}}\right\|_{H^{1 / 2}\left(\Gamma_{2}\right)}^{2} \mathrm{~d} s\right),
\end{aligned}
$$




$$
\begin{aligned}
& t^{2}\left\|\bar{u}_{t t}(t)\right\|^{2}+\int_{0}^{t} s^{2}\left\|\bar{u}_{s s}(s)\right\|_{1}^{2} \mathrm{~d} s \leqslant C\left(\left\|u_{0}\right\|_{2}^{2}+\sum_{j=0}^{2} \int_{0}^{t}\left\|\frac{\partial^{j} g_{2}}{\partial t^{j}}\right\|_{H^{1 / 2}\left(\Gamma_{2}\right)}^{2} \mathrm{~d} s\right), \\
& t^{i}\left\|\frac{\partial^{i} \bar{u}}{\partial t^{i}}\right\|_{2} \leqslant C\left\{\left\|u_{0}\right\|_{2}+\left(\sum_{j=0}^{i} \int_{0}^{t}\left\|\frac{\partial^{j} g_{2}}{\partial t^{j}}\right\|_{H^{1 / 2}\left(\Gamma_{2}\right)}^{2} \mathrm{~d} s\right)^{1 / 2}\right\}, \quad i=0,1, t \in J .
\end{aligned}
$$

\subsection{Finite volume element approximation}

Let $T_{h}$ be a quasi-uniform triangulation of $\Omega$ such that $\bar{\Omega}=\bigcup_{K \in T_{h}} K$, where $K$ is a closed triangle element. Let $N_{h}$ be the set of all nodes or vertices of $T_{h}$, i.e.,

$$
N_{h}=\left\{p: p \text { is a vertex of element } K \in T_{h} \text { and } p \in \bar{\Omega}\right\} .
$$

Further, we denote $N_{h}^{0}=N_{h} \cap \Omega$. For a vertex $x_{i} \in N_{h}$, let $\Pi(i)$ be the index set of those vertices that, along with $x_{i}$, are in some element of $T_{h}$.

For the triangulation $T_{h}$, we now introduce a dual mesh $T_{h}^{*}$ as follows: In each element $K \in T_{h}$ consisting of vertices $x_{i}, x_{j}$ and $x_{k}$, select a point $q \in K$, and select a point $x_{i j}$ by straight lines $\gamma_{i j, K}$. Then, for a vertex $x_{i}$, we let $V_{i}$ be the polygon whose edges are $\gamma_{i j, K}$ in which $x_{i}$ is a vertex of the element $K$. We call this $V_{i}$ a control volume centered at $x_{i}$. Further, we note that $\bigcup_{x_{i} \in N_{h}} V_{i}=\bar{\Omega}$. Thus, the dual mesh $T_{h}^{*}$ is then defined as the collection of these control volumes. A control volume centered at a vertex $x_{i}$ is given in Fig. 1.

We call the control volume mesh $T_{h}^{*}$ regular or quasi-uniform if there exists a positive constant $C>0$ such that

$$
C^{-1} h^{2} \leqslant \operatorname{meas}\left(V_{i}\right) \leqslant C h^{2} \text { for all } V_{i} \in T_{h}^{*},
$$

where $h$ is the maximum diameter of all elements $K \in T_{h}$.

There are various ways to introduce a regular dual mesh $T_{h}^{*}$ depending on the choices of the point $q$ in an element $K \in T_{h}$ and the points $x_{i j}$ on its edges. In this paper, we choose $q$ to be the barycenter of an element $K \in T_{h}$, and the points $x_{i j}$ are chosen to be the midpoints of the edges of $K$. In addition, if $T_{h}$ is locally regular, i.e., there is a constant $C$ such that

$$
C h_{K}^{2} \leqslant \operatorname{meas}(K) \leqslant h_{K}^{2},
$$

where $h_{K}=\operatorname{diam}(K)$ for all elements $K \in T_{h}$. Then the dual mesh $T_{h}^{*}$ is also locally regular. For the purpose of finite volume element approximation let $S_{h}$ be the linear finite element space defined on the triangulation $T_{h}$,

$$
S_{h}=\left\{v \in C(\Omega):\left.v\right|_{K} \text { is linear for all } K \in T_{h} \text { and }\left.v\right|_{\Gamma_{1}}=0\right\},
$$

and its dual volume element space $S_{h}^{*}$,

$$
S_{h}^{*}=\left\{v \in L^{2}(\Omega):\left.v\right|_{V} \text { is constant for all } V \in T_{h}^{*} \text { and }\left.v\right|_{\Gamma_{1}}=0\right\} .
$$
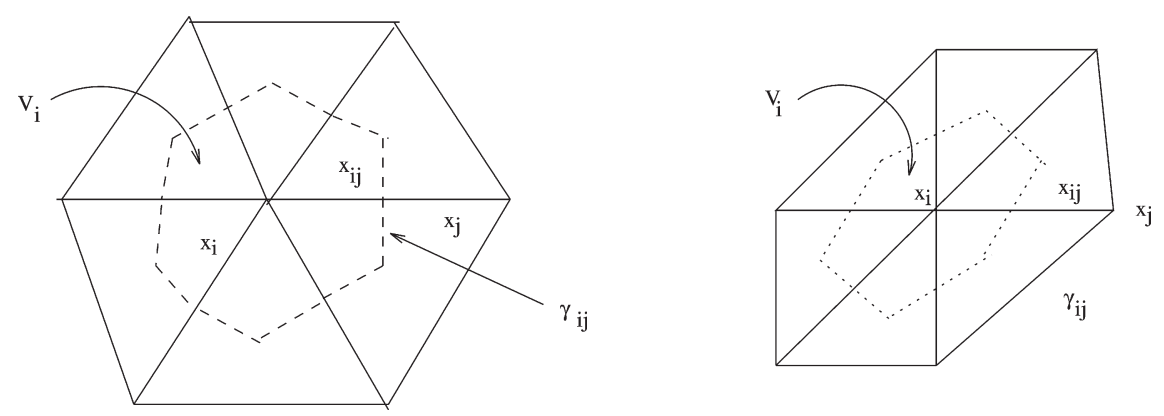

Fig. 1. Control volumes with barycenter as internal point and interface $\gamma_{i j}$ of $V_{i}$ and $V_{j}$. 
Obviously, $S_{h}=\operatorname{span}\left\{\phi_{i}(x): x_{i} \in N_{h}^{0}\right\}$ and $S_{h}^{*}=\operatorname{span}\left\{\chi_{i}(x): x_{i} \in N_{h}^{0}\right\}$, where $\phi_{i}$ are the standard nodal basis functions associated with the node $x_{i}$, and $\chi_{i}$ are the characteristic functions of the volume $V_{i}$. Let $I_{h}: C(\Omega) \rightarrow S_{h}$ and $I_{h}^{*}: C(\Omega) \rightarrow S_{h}^{*}$ be the usual interpolation operators, i.e.,

$$
I_{h} \bar{u}=\sum_{x_{i} \in N_{h}} \bar{u}_{i} \phi_{i}(x) \quad \text { and } \quad I_{h}^{*} \bar{u}=\sum_{x_{i} \in N_{h}} \bar{u}_{i} \chi_{i}(x),
$$

where $\bar{u}_{i}=\bar{u}\left(x_{i}\right)$.

The FVE approximation corresponding to (2.4) is defined to be the function $\bar{u}_{h}(t): \bar{J} \rightarrow S_{h}$ such that

$$
\left(\bar{u}_{h, t}, I_{h}^{*} \chi\right)+A_{\kappa}\left(\bar{u}_{h}, I_{h}^{*} \chi\right)=\left\langle\bar{g}_{2}, I_{h}^{*} \chi\right\rangle+\left(\bar{f}, I_{h}^{*} \chi\right)
$$

for all $\chi \in S_{h}$ with $\bar{u}_{h}(0)=u_{0, h}$, where $u_{0, h}$ is a suitable projection of $u_{0}$ onto $S_{h}$ to be defined later.

The bilinear forms $A_{\kappa}(\cdot, \cdot)$ in (2.6) is defined by

$$
A_{\kappa}(\bar{u}, w)=\sum_{x_{i} \in N_{h}}\left\{-w_{i} \int_{\partial V_{i}}(D(x) \nabla \bar{u}-\mathbf{v} \bar{u}) \cdot \mathbf{n} \mathrm{d} S_{x}+w_{i} \int_{V_{i}}(\lambda+\kappa) \bar{u} \mathrm{~d} x\right\}
$$

for $(\bar{u}, w) \in\left(\left(V \cap H^{2}\right) \cup S_{h}\right) \times S_{h}^{*}$, where $\mathbf{n}$ is the outer-normal vector of the involved integration domain. Note that when $(\bar{u}, w) \in V \times V$ the bilinear form $A_{\kappa}(\cdot, \cdot)$ is given by (2.5). Similarly, the FVE approximation to (2.1) is easily obtained by taking $\kappa=0$ in (2.6).

In order to describe features of the bilinear forms defined in (2.4) and (2.6) we define some discrete norms on $S_{h}$ and $S_{h}^{*}$,

$$
\begin{aligned}
& \left|u_{h}\right|_{0, h}^{2}=\left(u_{h}, u_{h}\right)_{0, h}, \quad\left|u_{h}\right|_{1, h}^{2}=\sum_{x_{i} \in N_{h}} \sum_{x_{j} \in \Pi(i)} \operatorname{meas}\left(V_{i}\right)\left(\left(u_{h i}-u_{h j}\right) / d_{i j}\right)^{2}, \\
& \left\|u_{h}\right\|_{1, h}^{2}=\left|u_{h}\right|_{0, h}^{2}+\left|u_{h}\right|_{1, h}^{2}, \quad\left\|u_{h}\right\|=\left(u_{h}, I_{h}^{*} u_{h}\right),
\end{aligned}
$$

where $\left(u_{h}, v_{h}\right)_{0, h}=\sum_{x_{i} \in N_{h}}$ meas $\left(V_{i}\right) u_{h i} v_{h i}=\left(I_{h}^{*} u_{h}, I_{h}^{*} v_{h}\right)$ and $d_{i j}=d\left(x_{i}, x_{j}\right)$ is the distance between $x_{i}$ and $x_{j}$. These norms are well defined for $u_{h} \in S_{h}^{*}$ as well and $\left\|u_{h}\right\|_{0, h}=\left\|u_{h}\right\|$.

Below, we state the equivalence of the discrete norms $|\cdot|_{0, h}$ and $\|\cdot\|_{1, h}$ with usual norms $\|\cdot\|$ and $\|\cdot\|_{1}$, respectively on $S_{h}$. Further, some properties of the bilinear forms are stated without proof. For a proof, we refer to $[9,10]$.

Lemma 2.3. There exist two positive constants $C_{1}$ and $C_{2}$ such that for all $v_{h} \in S_{h}$, we have

$$
\begin{aligned}
& C_{1}\left|v_{h}\right|_{0, h} \leqslant\left\|v_{h}\right\| \leqslant C_{2}\left|v_{h}\right|_{0, h}, \\
& C_{1}\left\|v_{h}\right\| \leqslant\left\|v_{h}\right\| \leqslant C_{2}\left\|v_{h}\right\|, \\
& C_{1}\left\|v_{h}\right\|_{1, h} \leqslant\left\|v_{h}\right\|_{1} \leqslant C_{2}\left\|v_{h}\right\|_{1, h} .
\end{aligned}
$$

Lemma 2.4. There exist positive constants $C$ and $c$ such that, for all $\phi_{h}, \psi_{h} \in S_{h}$, the boundedness property

$$
\left|A_{\kappa}\left(\phi_{h}, I_{h}^{*} \psi_{h}\right)\right| \leqslant C\left\|\phi_{h}\right\|_{1}\left\|\psi_{h}\right\|_{1}
$$

and the coercive property

$$
A_{\kappa}\left(\phi_{h}, I_{h}^{*} \phi_{h}\right) \geqslant c\left\|\phi_{h}\right\|_{1}^{2}
$$

hold true.

The following lemma gives the key feature of the bilinear forms in the finite volume element method. For a proof, see [10].

Lemma 2.5. Let $\phi \in\left(V \cap H^{2}\right) \cup S_{h}$. Then we have 


$$
\begin{aligned}
A_{\kappa}(\phi, \chi)-A_{\kappa}\left(\phi, I_{h}^{*} \chi\right)= & \sum_{K \in T_{h}} \int_{K}\{-\nabla \cdot(\mathcal{D} \nabla \phi-\mathbf{v} \phi)+(\lambda+\kappa) \phi\}\left(\chi-I_{h}^{*} \chi\right) \mathrm{d} x \\
& +\sum_{K \in T_{h}} \int_{\partial K}\{(\mathcal{D} \nabla \phi-\mathbf{v} \phi) \cdot \mathbf{n}\}\left(\chi-I_{h}^{*} \chi\right) \mathrm{d} S, \quad \forall \chi \in S_{h} .
\end{aligned}
$$

\section{Petrov-Ritz projection and related estimates}

Following [8,9], define the Petrov-Ritz projection $R_{h}: V \cap H^{2}(\Omega) \rightarrow S_{h}$ by

$$
A_{\kappa}\left(\bar{u}-R_{h} \bar{u}, I_{h}^{*} \chi\right)=0, \quad \forall \chi \in S_{h} .
$$

The following lemma prove to be convenient for obtaining $H^{1}$ and $L^{2}$ error estimates for the Petrov-Ritz projection.

Lemma 3.1. Assume that $\phi \in S_{h}$ and $\mathcal{D} \in W^{2, \infty}(\Omega)$. Then we have

$$
\left|A_{\kappa}(\phi, \chi)-A_{\kappa}\left(\phi, I_{h}^{*} \chi\right)\right| \leqslant C h\|\phi\|_{1}\|\chi\|_{1}, \quad \forall \chi \in S_{h} .
$$

Further, for $\phi \in V \cap H^{2}(\Omega)$, we have

$$
\left|A_{\kappa}(\phi, \chi)-A_{\kappa}\left(\phi, I_{h}^{*} \chi\right)\right| \leqslant C h\|\phi\|_{2}\|\chi\|_{1}, \quad \forall \chi \in S_{h} .
$$

Proof. Since the dual mesh is formed by the barycenters, we have for $\chi \in S_{h}$

$$
\int_{K}\left(\chi-I_{h}^{*} \chi\right) \mathrm{d} x=0 \quad \text { for all } K \in T_{h} .
$$

Thus, in view of Lemma 2.5, we have for $\phi, \chi \in S_{h}$

$$
\begin{aligned}
A_{\kappa}(\phi, \chi)-A_{\kappa}\left(\phi, I_{h}^{*} \chi\right)= & \sum_{K \in T_{h}} \int_{K}\{-\nabla \cdot(\mathcal{D} \nabla \phi-\mathbf{v} \phi)+(\lambda+\kappa) \phi\}\left(\chi-I_{h}^{*} \chi\right) \mathrm{d} \chi \\
& +\sum_{K \in T_{h}} \int_{\partial K}\left\{\left(\mathcal{D}-\overline{\mathcal{D}}_{K}\right)(\nabla \phi-\mathbf{v} \phi) \cdot \mathbf{n}\right\}\left(\chi-I_{h}^{*} \chi\right) \mathrm{d} S \\
:= & I_{1}+I_{2} .
\end{aligned}
$$

Here, $\overline{\mathcal{D}}_{K}$ is a function designed in a piecewise manner such that for any edge $E$ of a triangle $K \in T_{h}$ and $x \in E$, $\bar{D}_{K}(x)=D\left(x_{c}\right)$, where $x_{c}$ is the mid point of $E$. Noting that, for $\phi \in S_{h}, \nabla \phi$ is a constant on $K$, we have $\nabla \cdot(\mathcal{D} \nabla \phi)=$ $(\nabla \cdot \mathcal{D}) \nabla \phi$. Now, applying Cauchy-Schwarz's inequality and using the fact that $\left\|\chi-I_{h}^{*} \chi\right\| \leqslant C h\|\chi\|_{1}$, we obtain

$$
\left|I_{1}\right| \leqslant C h\|\phi\|_{1}\|\chi\|_{1} \text {. }
$$

Since $\left|\mathcal{D}(x)-\overline{\mathcal{D}}_{K}\right| \leqslant h\|\mathcal{D}\|_{1, \infty}$ and $\left\|\chi-I_{h}^{*} \chi\right\|_{L^{2}(\partial K)} \leqslant C h^{1 / 2}\|\chi\|_{1, K}$ (cf. [10]), the term $I_{2}$ is bounded by

$$
\left|I_{2}\right| \leqslant C h \sum_{K \in T_{h}} h^{1 / 2}\|\nabla \phi\|_{L^{2}(\partial K)}\|\chi\|_{1, K} \leqslant C h \sum_{K \in T_{h}}\|\phi\|_{1, K}\|\chi\|_{1, K} \leqslant C h\|\phi\|_{1}\|\chi\|_{1},
$$

where in the second inequality we have used the fact that $\nabla \phi$ is constant on $K$. Combine (3.2) and (3.4) to prove the first inequality.

Next, for $\phi \in V \cap H^{2}(\Omega)$, we have

$$
\left|I_{1}\right| \leqslant C h\|\phi\|_{2}\|\chi\|_{1} .
$$

For $I_{2}$, using the trace theorem [2], we obtain

$$
\left|I_{2}\right| \leqslant C h \sum_{K \in T_{h}} h^{1 / 2}\|\nabla \phi\|_{L^{2}(\partial K)}\left\|\chi-I_{h}^{*} \chi\right\|_{L^{2}(\partial K)} \leqslant C h\|\phi\|_{2}\|\chi\|_{1} .
$$


Combine (3.2), (3.5) and (3.6) to obtain the second inequality and this completes the proof.

Set $\rho=\bar{u}-R_{h} \bar{u}$. We now establish $H^{1}$-error estimate for $\rho$ and its temporal derivative.

Lemma 3.2. Let $\rho$ satisfy (3.1). Then we have

$$
\|\rho\|_{1} \leqslant C h\|\bar{u}\|_{2}, \quad\left\|\rho_{t}\right\|_{1} \leqslant C h\left\|\bar{u}_{t}\right\|_{2} .
$$

Proof. With $\phi_{h}=I_{h} \bar{u}-R_{h} \bar{u}$, we obtain using (3.1)

$$
\begin{aligned}
c\|\rho\|_{1}^{2} & \leqslant A_{\kappa}(\rho, \rho) \\
& =A_{\kappa}\left(\rho, \bar{u}-I_{h} \bar{u}\right)+A_{\kappa}\left(\rho, I_{h} \bar{u}-R_{h} \bar{u}\right) \\
& =A_{\kappa}\left(\rho, \bar{u}-I_{h} \bar{u}\right)+A_{\kappa}\left(\rho, \phi_{h}\right)-A_{\kappa}\left(\rho, I_{h}^{*} \phi_{h}\right) .
\end{aligned}
$$

An application of Lemma 3.1 yields

$$
\begin{aligned}
A_{\kappa}\left(\rho, \phi_{h}\right)-A_{\kappa}\left(\rho, I_{h}^{*} \phi_{h}\right) & =\left\{A_{\kappa}\left(\bar{u}, \phi_{h}\right)-A_{\kappa}\left(\bar{u}, I_{h}^{*} \phi_{h}\right)\right\}-\left\{A_{\kappa}\left(R_{h} \bar{u}, \phi_{h}\right)-A_{\kappa}\left(R_{h} \bar{u}, I_{h}^{*} \phi_{h}\right)\right\} \\
& \leqslant C h\left(\|\bar{u}\|_{2}+\|\bar{u}\|_{1}\right)\left\|\phi_{h}\right\|_{1} \\
& \leqslant C h\|\bar{u}\|_{2}\left(\|\rho\|_{1}+h\|\bar{u}\|_{2}\right),
\end{aligned}
$$

where in the last inequality we have used $\left\|\phi_{h}\right\|_{1} \leqslant C\left(h\|\bar{u}\|_{2}+\|\rho\|_{1}\right)$. Thus, we obtain

$$
c\|\rho\|_{1}^{2} \leqslant C h\|\bar{u}\|_{2}\|\rho\|_{1}+C h^{2}\|\bar{u}\|_{2}^{2} .
$$

Kickback the term $\|\rho\|_{1}$ to obtain the first inequality. For the second inequality, differentiate (3.1) with respect to time $t$ to have

$$
A_{\kappa}\left(\rho_{t}, I_{h}^{*} \chi\right)=0 .
$$

Then the rest of the proof follows in a similar fashion.

We shall prove the $L^{2}$ estimates of $\rho$ and its temporal derivatives in the following theorem.

Lemma 3.3. Let $\rho$ satisfy (3.1). Then we have

$$
\|\rho(t)\| \leqslant C h^{2}\|\bar{u}\|_{2}, \quad\left\|\rho_{t}(t)\right\| \leqslant C h^{2}\left\|\bar{u}_{t}\right\|_{2} .
$$

Proof. The proof will proceed by duality argument. Let $\psi \in H^{2}(\Omega) \cap H_{0}^{1}(\Omega)$ be the solution of

$$
A_{\kappa}^{*} \psi=\rho \quad \text { in } \Omega, \quad \psi=0 \quad \text { on } \partial \Omega,
$$

where $A_{\kappa}^{*}$ is the formal adjoint of $A_{\kappa}$. The solution $\psi$ satisfies the following regularity estimate

$$
\|\psi\|_{2} \leqslant C\|\rho\| .
$$

Multiplying (3.8) by $\rho$ and then taking $L^{2}$ inner-product over $\Omega$, we obtain

$$
\|\rho\|^{2}=A_{\kappa}\left(\rho, \psi-I_{h} \psi\right)+A_{\kappa}\left(\rho, I_{h} \psi\right)=I_{1}+I_{2} .
$$

Using Lemma 3.2, $I_{1}$ is bounded as

$$
\left|I_{1}\right| \leqslant C h^{2}\|\bar{u}\|_{2}\|\psi\|_{2} .
$$

Following the line of arguments of [10, Theorem 3.5], the term $I_{2}$ is bounded as

$$
\left|I_{2}\right| \leqslant C h^{2}\|u\|_{2}\|\psi\|_{2}
$$

which combine with (3.10), (3.11) and (3.9) completes the proof. 


\section{Error estimates for the spatially discrete scheme}

In this section, the error analysis for the spatially discrete FVE approximation will be carried out. For homogeneous problem, optimal order error estimates are established in $L^{2}$ and $H^{1}$ norms when $u_{0} \in H^{2} \cap V$. In addition, a quasioptimal order error estimate in $L^{\infty}$ norm is proved in an interior sub-domain away from the corners.

As usual we split the error $e=\bar{u}-\bar{u}_{h}$ as

$$
e=\left(\bar{u}-R_{h} \bar{u}\right)+\left(R_{h} \bar{u}-\bar{u}_{h}\right)=\rho+\theta .
$$

Since the estimates of $\rho$ are already known, it is enough to have estimates for $\theta$.

Using (2.6), an equation of the form (2.6) with $u_{h}$ replaced by $u$ and (3.1), it is easy to verify that $\theta$ satisfies an error equation

$$
\left(\theta_{t}, I_{h}^{*} \chi\right)+A_{\kappa}\left(\theta, I_{h}^{*} \chi\right)=-\left(\rho_{t}, I_{h}^{*} \chi\right), \quad \forall \chi \in S_{h} .
$$

Define $\hat{\theta}(t)=\int_{0}^{t} \theta(s) \mathrm{d} s$. Then, clearly $\hat{\theta}(0)=0$ and $\hat{\theta}_{t}=\theta$. We shall prove a sequence of lemmas which lead to the desired result.

Lemma 4.1. Assume that $\bar{u}_{h}(0)=R_{h} u_{0}$. There is a positive constant $C$ independent of $h$ such that

$$
\int_{0}^{t}\|\theta(s)\|^{2} \mathrm{~d} s+\|\hat{\theta}(t)\|_{1}^{2} \leqslant C\left(t\|\rho(0)\|^{2}+\int_{0}^{t}\|\rho(s)\|^{2} \mathrm{~d} s\right) .
$$

Proof. Integrate (4.1) from 0 to $t$ and use the fact $\theta(0)=0$ to have

$$
\left(\theta, I_{h}^{*} \chi\right)+A_{\kappa}\left(\hat{\theta}, I_{h}^{*} \chi\right)=-\left(\rho(t), I_{h}^{*} \theta\right)+\left(\rho(0), I_{h}^{*} \theta\right) .
$$

Choose $\chi=\theta$ in (4.2) to obtain

$$
\begin{aligned}
\|\theta\|^{2}+\frac{1}{2} \frac{\mathrm{d}}{\mathrm{d} t}\left\{A_{\kappa}(\hat{\theta}, \hat{\theta})\right\} & =-\left(\rho, I_{h}^{*} \theta\right)+\left(\rho(0), I_{h}^{*} \theta\right)+\left\{A_{\kappa}(\hat{\theta}, \theta)-A_{\kappa}\left(\hat{\theta}, I_{h}^{*} \theta\right)\right\} \\
& \leqslant(\|\rho\|+\|\rho(0)\|)\|\theta\|+C\|\hat{\theta}\|_{1}\|\theta\|,
\end{aligned}
$$

where in the last step, we have used the fact that (cf. [6, Lemma 4.1])

$$
\left|A_{\kappa}(\hat{\theta}, \theta)-A_{\kappa}\left(\hat{\theta}, I_{h}^{*} \theta\right)\right| \leqslant C\|\hat{\theta}\|_{1}\|\theta\| .
$$

Integrating (4.3) from 0 to $t$ and using Lemma 2.3, we obtain

$$
\int_{0}^{t}\|\theta(s)\|^{2} \mathrm{~d} s+\|\hat{\theta}(t)\|_{1}^{2} \leqslant C \int_{0}^{t}\left(\|\rho\|^{2}+\|\rho(0)\|^{2}\right) \mathrm{d} s+\frac{1}{2} \int_{0}^{t}\|\theta\|^{2} \mathrm{~d} s+\int_{0}^{t}\|\hat{\theta}\|_{1}^{2} \mathrm{~d} s .
$$

Kickback the term $\frac{1}{2} \int_{0}^{t}\|\theta\|^{2} \mathrm{~d} s$ and then apply Gronwall's lemma to complete the rest of the proof.

Lemma 4.2. Let $\theta$ satisfy (4.1) with $\bar{u}_{h}(0)=R_{h} u_{0}$. Then there is a positive constant $C$ independent of $h$ such that

$$
t\|\theta(t)\|^{2}+\int_{0}^{t} s\|\theta(s)\|_{1}^{2} \mathrm{~d} s \leqslant C\left(t\|\rho(0)\|^{2}+\int_{0}^{t}\left\{\|\rho(s)\|^{2}+s^{2}\left\|\rho_{s}(s)\right\|^{2}\right\} \mathrm{d} s\right) .
$$

Proof. Set $\chi=t \theta$ in (4.1). Then using the symmetry of $\left(\psi, I_{h}^{*} \chi\right), \psi, \chi \in S_{h}$ on $S_{h}$, we obtain

$$
\frac{1}{2} \frac{\mathrm{d}}{\mathrm{d} t}\left\{t\|\theta\|^{2}\right\}+t A_{\kappa}\left(\theta, I_{h}^{*} \theta\right) \leqslant\|\theta\|^{2}+t\left\|\rho_{t}\right\|\left\|I_{h}^{*} \theta\right\| .
$$

Integrating from 0 to $t$ and using the weak coercivity in Lemma 2.4, it now leads to 


$$
\frac{1}{2} t\|\theta(t)\|^{2}+\int_{0}^{t} s\|\theta(s)\|_{1}^{2} \mathrm{~d} s \leqslant C \int_{0}^{t}\|\theta\|^{2} \mathrm{~d} s+\int_{0}^{t} s\left\|\rho_{t}\right\|\|\theta\| \mathrm{d} s .
$$

Apply Young's inequality to have

$$
t\|\theta(t)\|^{2}+\int_{0}^{t} s\|\theta(s)\|_{1}^{2} \mathrm{~d} s \leqslant C\left(\int_{0}^{t}\left\{\|\theta\|^{2}+s^{2}\left\|\rho_{s}\right\|^{2}\right\} \mathrm{d} s\right) .
$$

Finally, use Lemma 4.1 to complete the rest of the proof.

Lemma 4.3. Let the hypotheses in Lemma 4.2 hold true. Then there is a positive constant $C$ independent of $h$ such that

$$
\int_{0}^{t} s^{2}\|\theta(s)\|^{2} \mathrm{~d} s+t^{2}\|\theta(t)\|_{1}^{2} \leqslant C\left(t\|\rho(0)\|^{2}+\int_{0}^{t}\left\{\|\rho(s)\|^{2}+s^{2}\left\|\rho_{s}(s)\right\|^{2}\right\} \mathrm{d} s\right) .
$$

Proof. Choose $\chi=t^{2} \theta_{t}$ in (4.1) to have

$$
t^{2}\left\|\theta_{t}(t)\right\|^{2}+\frac{1}{2} \frac{\mathrm{d}}{\mathrm{d} t}\left\{t^{2} A_{\kappa}(\theta, \theta)\right\}=-t^{2}\left(\rho_{t}, I_{h}^{*} \theta_{t}\right)+t A_{\kappa}(\theta, \theta)+t^{2}\left\{A_{\kappa}\left(\theta, \theta_{t}\right)-A_{\kappa}\left(\theta, I_{h}^{*} \theta_{t}\right)\right\} .
$$

It follows from [6, Lemma 4.1] that

$$
\left|A_{\kappa}\left(\theta, \theta_{t}\right)-A_{\kappa}\left(\theta, I_{h}^{*} \theta_{t}\right)\right| \leqslant C\|\theta\|_{1}\left\|\theta_{t}\right\| .
$$

Now integrate (4.4) from 0 to $t$. Then apply Lemmas 2.3 and 2.4 and standard kickback argument to obtain

$$
\int_{0}^{t} s^{2}\left\|\theta_{s}(s)\right\|^{2} \mathrm{~d} s+t^{2}\|\theta(t)\|_{1}^{2} \leqslant C \int_{0}^{t} s\|\theta(s)\|_{1}^{2} \mathrm{~d} s+C \int_{0}^{t} s^{2}\left\|\rho_{s}\right\|^{2} \mathrm{~d} s+C \int_{0}^{t} s^{2}\|\theta\|_{1}^{2} \mathrm{~d} s .
$$

Finally, apply Lemma 4.2 and Gronwall's lemma to complete the proof.

The main results of this section is given in the following theorems.

Theorem 4.1. Let $\tilde{u}$ satisfy (1.4) with $f=0$, and let $\tilde{u}_{h}$ be its FVE approximation. Then, for $u_{0} \in H^{2} \cap V, \frac{\partial^{j} g_{2}}{\partial t^{j}} \in$ $H^{1 / 2}\left(\Gamma_{2}\right)(j=0,1,2)$ and $\bar{u}_{h}(0)=R_{h} u_{0}$, we have

$$
\left\|\tilde{u}(t)-\tilde{u}_{h}(t)\right\|_{1} \leqslant C h t^{-1 / 2}\left\{\left\|u_{0}\right\|_{2}+\left(\sum_{j=0}^{2} \int_{0}^{t}\left\|\frac{\partial^{j} g_{2}}{\partial t^{j}}\right\|_{H^{1 / 2}\left(\Gamma_{2}\right)}^{2} \mathrm{~d} s\right)^{1 / 2}\right\}
$$

and

$$
\left\|\tilde{u}(t)-\tilde{u}_{h}(t)\right\| \leqslant C h^{2}\left\{\left\|u_{0}\right\|_{2}+\left(\sum_{j=0}^{2} \int_{0}^{t}\left\|\frac{\partial^{j} g_{2}}{\partial t^{j}}\right\|_{H^{1 / 2}\left(\Gamma_{2}\right)}^{2} \mathrm{~d} s\right)^{1 / 2}\right\}
$$

hold true for $t \in J$.

Proof. By triangle inequality, we have

$$
\left\|\bar{u}(t)-\bar{u}_{h}(t)\right\|_{1} \leqslant\|\rho(t)\|_{1}+\|\theta(t)\|_{1} .
$$

From Lemma 4.3, we obtain 


$$
\begin{aligned}
t\|\theta\|_{1} & \leqslant C\left(t\left\|R_{h} u_{0}-u_{0}\right\|^{2}+\int_{0}^{t}\left\{\|\rho(s)\|^{2}+s^{2}\left\|\rho_{s}(s)\right\|^{2}\right\} \mathrm{d} s\right)^{1 / 2} \\
& \leqslant C h\left(t\left\|u_{0}\right\|_{2}^{2}+\int_{0}^{t}\left\{\|\bar{u}\|_{2}^{2}+s^{2}\left\|\bar{u}_{s}\right\|_{2}^{2}\right\} \mathrm{d} s\right)^{1 / 2} .
\end{aligned}
$$

In view of Lemma 2.2, it now follows that

$$
t\|\theta\|_{1} \leqslant C h t^{1 / 2}\left\{\left\|u_{0}\right\|_{2}+\left(\sum_{j=0}^{2} \int_{0}^{t}\left\|\frac{\partial^{j} g_{2}}{\partial t^{j}}\right\|_{H^{1 / 2}\left(\Gamma_{2}\right)}^{2} \mathrm{~d} s\right)^{1 / 2}\right\}
$$

and this together with Lemmas 3.2, 2.2 and the identity

$$
\bar{u}-\bar{u}_{h}=e^{-\kappa t}\left(\tilde{u}-\tilde{u}_{h}\right)
$$

yield the first inequality. Similarly, for the second inequality, we use Lemmas 3.3, 4.2, a priori estimates in Lemma 2.2 and the identity (4.6). This completes the rest of the proof.

We shall close this section by showing a quasi-optimal order error estimate in maximum norm in an interior domain $\Omega_{0} \subset \Omega$ with $\bar{\Omega}_{0}$ not containing any vertex of $\Omega$.

Theorem 4.2. Let $\Omega_{0} \subset \Omega$ be such that $\bar{\Omega}_{0}$ does not contain any vertex of $\Omega$. Further, let $\tilde{u}$ satisfy (1.4) with $f=0$, and let $\tilde{u}_{h}$ be its FVE approximation. Assume that $u_{0} \in H^{2} \cap V, \frac{\partial^{j} g_{2}}{\partial t^{j}} \in H^{1 / 2}\left(\Gamma_{2}\right)(j=0,1,2)$ and $\bar{u}_{h}(0)=R_{h} u_{0}$. Then there is a positive constant $C$ such that

$$
\left\|\tilde{u}(t)-\tilde{u}_{h}(t)\right\|_{L^{\infty}\left(\Omega_{0}\right)} \leqslant C t^{-1} h^{2} \log \frac{1}{h}\left\{\left\|u_{0}\right\|_{2}+\left(\sum_{j=0}^{2} \int_{0}^{t}\left\|\frac{\partial^{j} g_{2}}{\partial t^{j}}\right\|_{H^{1 / 2}\left(\Gamma_{2}\right)}^{2} \mathrm{~d} s\right)^{1 / 2}\right\}, \quad t \in J .
$$

Proof. By triangle inequality, we have

$$
\left\|\bar{u}(t)-\bar{u}_{h}(t)\right\|_{L^{\infty}\left(\Omega_{0}\right)} \leqslant\|\theta(t)\|_{L^{\infty}\left(\Omega_{0}\right)}+\|\rho(t)\|_{L^{\infty}\left(\Omega_{0}\right)} .
$$

Recall that $S_{h}$ is the linear finite element space and triangulation is quasi-uniform, we thus have (cf. [20, Chapter 5])

$$
\|\theta(t)\|_{L^{\infty}} \leqslant C\left(\log \frac{1}{h}\right)^{1 / 2}\|\theta(t)\|_{1},
$$

and hence, using (4.5), it now follows that

$$
\|\theta(t)\|_{L^{\infty}\left(\Omega_{0}\right)} \leqslant C h^{2} t^{-1 / 2} \log \frac{1}{h}\left[\left\|u_{0}\right\|_{2}+\left(\sum_{j=0}^{2} \int_{0}^{t}\left\|\frac{\partial^{j} g_{2}}{\partial t^{j}}\right\|_{H^{1 / 2}\left(\Gamma_{2}\right)}^{2} \mathrm{~d} s\right)^{1 / 2}\right] .
$$

Thus, the first term in (4.7) is bounded as desired. It now remains to bound $\|\rho\|_{L^{\infty}\left(\Omega_{0}\right)}$. Let $\Omega_{2}$ and $\Omega_{3}$ be domains with $\Omega_{1} \subset \Omega_{2} \subset \Omega_{3} \subset \Omega$ and smooth boundaries. Further, let $\Omega_{3}$ does not contain any corner of $\Omega$ and the distances between $\partial \Omega_{3} \cap \Omega, \partial \Omega_{2} \cap \Omega$, and $\partial \Omega_{1} \cap \Omega$ are positive. Let $\omega$ be a smooth function such that $\left.\omega\right|_{\Omega_{2}}=1$ and $\left.\omega\right|_{\partial \Omega_{3} \cap \Omega}=$ 0 . It is well known that (cf. [6])

$$
\|\rho(t)\|_{L^{\infty}\left(\Omega_{0}\right)} \leqslant C h^{2} \log \frac{1}{h}\|\bar{u}(t)\|_{W^{2, \infty}\left(\Omega_{2}\right)}+C\|\rho(t)\| .
$$

Since the term $\|\rho\|$ is bounded as desired by Lemma 3.3, it now remains to bound the first term $\|\bar{u}(t)\|_{W^{2, \infty}\left(\Omega_{2}\right)}$. Using Sobolev inequality and elliptic regularity estimate in $\Omega_{3}$ (recall that $\partial \Omega_{3}$ is smooth), we obtain, with $\overline{\bar{u}}=\omega \bar{u}$,

$$
\begin{aligned}
\|\bar{u}\|_{W^{2, \infty}\left(\Omega_{2}\right)} & \leqslant C\|\bar{u}\|_{W^{3, p}\left(\Omega_{2}\right)} \leqslant C\|\overline{\bar{u}}\|_{W^{3, p}\left(\Omega_{3}\right)} \leqslant C\left\|A_{\kappa} \overline{\bar{u}}\right\|_{W^{1, p}\left(\Omega_{3}\right)} \\
& \leqslant C\left(\left\|A_{\kappa} \bar{u}\right\|_{W^{1, p}}+\|\bar{u}\|_{W^{2, p}}\right) \leqslant C\left\|A_{\kappa} \bar{u}\right\|_{W^{1, p}},
\end{aligned}
$$


where $2<p<2 /(2-\beta)$ with $1<\beta$. In the last inequality, we have used the following regularity estimate (cf. [14, Theorem 5.2.7])

$$
\|\bar{u}\|_{W^{2, p}} \leqslant C\left\|A_{\kappa} \bar{u}\right\|_{L^{p}} .
$$

Using (2.3) with $f=0$, Sobolev inequality and Lemma 2.2, it now follows that

$$
\begin{aligned}
\left\|A_{\kappa} \bar{u}\right\|_{W^{1, p}} & \leqslant C\left\|\bar{u}_{t}\right\|_{W^{1, p}} \leqslant C\left\|\bar{u}_{t}\right\|_{H^{2}} \\
& \leqslant C\left\|\bar{u}_{t t}\right\| \leqslant C t^{-1}\left[\left\|u_{0}\right\|_{2}+\left(\sum_{j=0}^{2} \int_{0}^{t}\left\|\frac{\partial^{j} g_{2}}{\partial t^{j}}\right\|_{H^{1 / 2}\left(\Gamma_{2}\right)}^{2} \mathrm{~d} s\right)^{1 / 2}\right]
\end{aligned}
$$

for $t \in J$. Combine (4.7)-(4.11) with (4.6) to complete the rest of the proof.

\section{Discrete-in-time scheme}

In this section, based on backward Euler method we shall discuss fully discrete approximations to (2.6). While optimal order error estimates are obtained in $L^{2}$ and $H^{1}$ norms, a quasi-optimal order error estimate in $L^{\infty}$ norm is established in any sub-domain away from the corners.

Let $k>0$ be the time step and $t_{n}=n k$ with $T=N k$. For any continuous function $\psi(t)$, set $\psi^{n}=\psi\left(t_{n}\right)$ and $\bar{\partial}_{t} \psi^{n}=k^{-1}\left(\psi^{n}-\psi^{n-1}\right)$. For $\phi \in S_{h}$, define $\|\phi\|_{-j, h}$ as

$$
\|\phi\|_{-j, h}=\sup _{g \in S_{h}} \frac{\left(\phi, I_{h}^{*} g\right)}{\|g\|_{j}}, \quad j=0,1 .
$$

The discrete in time Euler scheme is to seek a function $U^{n}, n=1,2, \ldots, N$ satisfying

$$
\left(\bar{\partial}_{t} U^{n}, I_{h}^{*} \chi\right)+A_{\kappa}\left(U^{n}, I_{h}^{*} \chi\right)=\left\langle\bar{g}_{2}, I_{h}^{*} \chi\right\rangle+\left(\bar{f}^{n}, I_{h}^{*} \chi\right) \quad \forall \chi \in S_{h},
$$

with given $U^{0}=R_{h} u_{0}$.

Set $U^{n}=e^{-\kappa t_{n}} \widetilde{U}^{n}$, where $\widetilde{U}^{n}$ is the backward Euler approximation to (1.4) which may be obtained by putting $\kappa=0$ in (5.1). Note that if $U^{n}$ 's are known then we can easily compute $\widetilde{U}^{n}$ 's.

Denote $\eta^{n}=U^{n}-\bar{u}_{h}^{n}$. Then, from (2.6) and (5.1), $\eta^{n}$ satisfies

$$
\left(\bar{\partial}_{t} \eta^{n}, I_{h}^{*} \chi\right)+A_{\kappa}\left(\eta^{n}, I_{h}^{*} \chi\right)=\left(\tau^{n}, I_{h}^{*} \chi\right), \quad \chi \in S_{h}
$$

with $\eta^{0}=0$, where $\tau^{n}=\bar{u}_{h t}^{n}-\bar{\partial}_{t} \bar{u}_{h}^{n}$.

Lemma 5.1. Let $\eta^{n}$ satisfy (5.2) and $\bar{u}_{h}(0)=R_{h} u_{0}$. Then there exists a constant $C$ independent of $k$ such that

$$
\left\|\eta^{n}\right\|^{2}+k \sum_{j=1}^{n}\left\|\eta^{j}\right\|_{1}^{2} \leqslant C k^{2}\left(\left\|u_{0}\right\|_{2}^{2}+\sum_{j=0}^{1} \int_{0}^{t}\left\|\frac{\partial^{j} g_{2}}{\partial t^{j}}\right\|_{H^{1 / 2}\left(\Gamma_{2}\right)}^{2} \mathrm{~d} s\right) .
$$

Proof. Taking $\chi=\eta^{n}$ in (5.2) and using the symmetry of $\left(\chi, I_{h}^{*} \psi\right), \chi, \psi \in S_{h}$ on $S_{h}$, and the identity $\left(\bar{\partial}_{t} \eta^{n}, I_{h}^{*} \eta^{n}\right)=$ $\frac{1}{2} \bar{\partial}_{t}\left\{\left\|\eta^{n}\right\|^{2}\right\}+\frac{k}{2}\left\|\bar{\partial}_{t} \eta^{n}\right\|^{2}$ leads to

$$
\frac{1}{2} \bar{\partial}_{t}\left\{\left\|\left|\eta^{n} \|\right|^{2}\right\}+A_{\kappa}\left(\eta^{n}, I_{h}^{*} \eta^{n}\right)+\frac{k}{2}\left\|\bar{\partial}_{t} \eta^{n}\right\|^{2}=\left(\tau^{n}, I_{h}^{*} \eta^{n}\right) \leqslant\left\|\tau^{n}\right\|_{-1, h}\left\|\eta^{n}\right\|_{1} .\right.
$$

Apply Young's inequality and kickback the term $\left\|\eta^{n}\right\|_{1}^{2}$ to obtain

$$
\frac{1}{2} \bar{\partial}_{t}\left\{\left\|\eta^{n}\right\|^{2}\right\}+\left\|\eta^{n}\right\|_{1}^{2} \leqslant C\left\|\tau^{n}\right\|_{-1, h}^{2}
$$

Summing over $n$ from 1 to $m$ and using Lemma 2.3, it now leads to

$$
\left\|\eta^{m}\right\|^{2}+k \sum_{n=1}^{m}\left\|\eta^{n}\right\|_{1}^{2} \leqslant C\left(\left\|\eta^{0}\right\|^{2}+k \sum_{n=1}^{m}\left\|\tau^{n}\right\|_{-1, h}^{2}\right) .
$$


Since $\eta^{0}=0$, it now remains to estimate the second term on the right. We write $\tau^{j}$ as

$$
\tau^{j}=\frac{1}{k} \int_{t_{j-1}}^{t_{j}}\left(s-t_{j-1}\right) \bar{u}_{h s s}(s) \mathrm{d} s
$$

and hence,

$$
\left\|\tau^{j}\right\|_{-1, h}^{2} \leqslant k \int_{t_{j-1}}^{t_{j}}\left\|\bar{u}_{h s s}(s)\right\|_{-1, h}^{2} \mathrm{~d} s .
$$

Differentiating (2.6) with respect to time $t$, we obtain for $f=0$

$$
\left(\bar{u}_{h t t}(t), I_{h}^{*} \chi\right)=-A_{\kappa}\left(\bar{u}_{h t}(t), I_{h}^{*} \chi\right)+\left(\frac{\partial \bar{g}_{2}}{\partial t}, I_{h}^{*} \chi\right)
$$

and this implies

$$
\left\|\bar{u}_{h t t}(t)\right\|_{-1, h} \leqslant C\left(\left\|\bar{u}_{h t}(t)\right\|_{1}+\sum_{j=0}^{1}\left\|\frac{\partial^{j} g_{2}}{\partial t^{j}}\right\|_{H^{1 / 2}\left(\Gamma_{2}\right)}\right) .
$$

Applying Lemma 2.2 at the discrete level, it now follows that

$$
k \sum_{j=1}^{n}\left\|\tau^{j}\right\|_{-1, h}^{2} \leqslant C k^{2} \int_{0}^{t_{n}}\left\{\left\|\bar{u}_{h s}(s)\right\|_{1}^{2}+\sum_{j=0}^{1}\left\|\frac{\partial^{j} g_{2}}{\partial t^{j}}\right\|_{H^{1 / 2}\left(\Gamma_{2}\right)}^{2}\right\} \mathrm{d} s \leqslant C k^{2}\left[\left\|u_{0}\right\|_{2}^{2}+\sum_{j=0}^{1} \int_{0}^{t_{n}}\left\|\frac{\partial^{j} g_{2}}{\partial t^{j}}\right\|_{H^{1 / 2}\left(\Gamma_{2}\right)}^{2} \mathrm{~d} s\right],
$$

and this completes the rest of the proof.

Lemma 5.2. Let the hypotheses in Lemma 5.1 hold true. Then there is a constant $C$ independent of $k$ such that

$$
k \sum_{j=1}^{n} t_{j}\left\|\bar{\partial}_{t} \eta^{j}\right\|^{2}+t_{n}\left\|\eta^{n}\right\|_{1}^{2} \leqslant C k^{2}\left(\left\|u_{0}\right\|_{2}^{2}+\sum_{j=0}^{1} \int_{0}^{t_{n}}\left\|\frac{\partial^{j} g_{2}}{\partial t^{j}}\right\|_{H^{1 / 2}\left(\Gamma_{2}\right)}^{2} \mathrm{~d} s\right) .
$$

Taking $\chi=t_{n} \bar{\partial}_{t} \eta^{n}$ in (5.2) and using identity

$$
t_{n} A_{\kappa}\left(\eta^{n}, \bar{\partial}_{t} \eta^{n}\right)=\frac{1}{2} \bar{\partial}_{t}\left\{t_{n} A_{\kappa}\left(\eta^{n}, \eta^{n}\right)\right\}+\frac{k}{2} t_{n} A_{\kappa}\left(\bar{\partial}_{t} \eta^{n}, \bar{\partial}_{t} \eta^{n}\right)-\frac{1}{2} A_{\kappa}\left(\eta^{n-1}, \eta^{n-1}\right)
$$

we obtain

$$
\begin{aligned}
& t_{n}\left\|\bar{\partial}_{t} \eta^{n}\right\|^{2}+\frac{1}{2} \bar{\partial}_{t}\left\{t_{n} A_{\kappa}\left(\eta^{n}, \eta^{n}\right)\right\}+\frac{k}{2} t_{n} A_{\kappa}\left(\bar{\partial}_{t} \eta^{n}, \bar{\partial}_{t} \eta^{n}\right) \\
& \quad=t_{n}\left(\tau^{n}, \bar{\partial}_{t} \eta^{n}\right)+\frac{1}{2} A_{\kappa}\left(\eta^{n-1}, \eta^{n-1}\right)+t_{n}\left\{A_{\kappa}\left(\eta^{n}, \bar{\partial}_{t} \eta^{n}\right)-A_{\kappa}\left(\eta^{n}, I_{h}^{*} \bar{\partial}_{t} \eta^{n}\right)\right\} \\
& \quad \leqslant t_{n}\left\|\tau^{n}\right\|\left\|\bar{\partial}_{t} \eta^{n}\right\|+C\left\|\eta^{n-1}\right\|_{1}^{2}+C t_{n}\left\|\eta^{n}\right\|_{1}\left\|\bar{\partial}_{t} \eta^{n}\right\| .
\end{aligned}
$$

Summing over $n$ from 1 to $m$ and using standard kickback arguments to have

$$
k \sum_{n=1}^{m} t_{n}\left\|\bar{\partial}_{t} \eta^{n}\right\|^{2}+t_{m}\left\|\eta^{m}\right\|_{1}^{2} \leqslant C k \sum_{n=1}^{m} t_{n}\left\|\tau^{n}\right\|^{2}+C k \sum_{n=1}^{m-1}\left\|\eta^{n}\right\|_{1}^{2}+C k \sum_{n=1}^{m} t_{n}\left\|\eta^{n}\right\|_{1}^{2} .
$$

From (5.3), we note that

$$
k \sum_{n=1}^{m} t_{n}\left\|\tau^{n}\right\|^{2} \leqslant \sum_{n=1}^{m} \int_{t_{n-1}}^{t_{n}} t_{n}\left(s-t_{n-1}\right)^{2}\left\|\bar{u}_{h s s}\right\|^{2} \mathrm{~d} s
$$


Since $\left(s-t_{n-1}\right)^{2} t_{n} \leqslant C k^{2} s$ for $s \in\left[t_{n-1}, t_{n}\right]$, we have

$$
k \sum_{n=1}^{m} t_{n}\left\|\tau^{n}\right\|^{2} \leqslant C k^{2} \int_{0}^{t_{n}} s\left\|\bar{u}_{h s s}\right\|^{2} \mathrm{~d} s \leqslant C k^{2}\left(\left\|u_{0}\right\|_{2}^{2}+\sum_{j=0}^{1} \int_{0}^{t_{n}}\left\|\frac{\partial^{j} g_{2}}{\partial t^{j}}\right\|_{H^{1 / 2}\left(\Gamma_{2}\right)}^{2} \mathrm{~d} s\right) .
$$

Now using Lemma 5.1, we obtain, for sufficiently small $k$,

$$
k \sum_{n=1}^{m} t_{n}\left\|\bar{\partial}_{t} \eta^{n}\right\|^{2}+t_{m}\left\|\eta^{m}\right\|_{1}^{2} \leqslant C k^{2}\left(\left\|u_{0}\right\|_{2}^{2}+\sum_{j=0}^{1} \int_{0}^{t_{n}}\left\|\frac{\partial^{j} g_{2}}{\partial t^{j}}\right\|_{H^{1 / 2}\left(\Gamma_{2}\right)}^{2} \mathrm{~d} s\right)+C k \sum_{n=1}^{m-1} t_{n}\left\|\eta^{n}\right\|_{1}^{2} .
$$

Finally, apply discrete version of Gronwall's lemma to completes the proof.

Theorem 5.1. Let $\tilde{u}$ be the exact solution of (1.4) with $f=0$, and $\widetilde{U}^{n}$ be its backward Euler approximation at $t=t_{n}$. Then there is a constant $C$ independent of $h$ and $k$ such that, for $n=1,2, \ldots, N$, we have

$$
\left\|\tilde{U}^{n}-\tilde{u}\left(t_{n}\right)\right\|_{1} \leqslant C t_{n}^{-1 / 2}(h+k)\left\{\left\|u_{0}\right\|_{2}+\left(\sum_{j=0}^{2} \int_{0}^{t_{n}}\left\|\frac{\partial^{j} g_{2}}{\partial t^{j}}\right\|_{H^{1 / 2}\left(\Gamma_{2}\right)}^{2} \mathrm{~d} s\right)^{1 / 2}\right\}
$$

and

$$
\left\|\widetilde{U}^{n}-\tilde{u}\left(t_{n}\right)\right\| \leqslant C\left(h^{2}+k\right)\left\{\left\|u_{0}\right\|_{2}+\left(\sum_{j=0}^{2} \int_{0}^{t_{n}}\left\|\frac{\partial^{j} g_{2}}{\partial t^{j}}\right\|_{H^{1 / 2}\left(\Gamma_{2}\right)}^{2} \mathrm{~d} s\right)^{1 / 2}\right\} .
$$

Proof. We write $\widetilde{U}^{n}-\tilde{u}\left(t_{n}\right)$ as

$$
\tilde{U}^{n}-\tilde{u}\left(t_{n}\right)=e^{\kappa t_{n}}\left(U^{n}-\bar{u}_{h}\left(t_{n}\right)\right)-\left(\tilde{u}\left(t_{n}\right)-\tilde{u}_{h}\left(t_{n}\right)\right)=e^{\kappa t_{n}} \eta^{n}-\left(\tilde{u}\left(t_{n}\right)-\tilde{u}_{h}\left(t_{n}\right)\right) .
$$

Now, combine Lemmas 5.2 and 5.1 with Theorem 4.1 to obtain the desired estimates and this completes the rest of the proof.

Theorem 5.2. Let $\Omega_{0} \subset \Omega$ be such that $\bar{\Omega}_{0}$ does not contain any vertex of $\Omega$. Further, let $\tilde{u}$ be the exact solution of (1.4) with $f=0$, and $\widetilde{U}^{n}$ be its backward Euler approximation at $t=t_{n}$. Then there is a positive constant $C$ such that

$$
\left\|\tilde{U}^{n}-\tilde{u}\left(t_{n}\right)\right\|_{L_{\infty}\left(\Omega_{0}\right)} \leqslant C t_{n}^{-1} \log \frac{1}{h}\left(h^{2}+k\right)\left\{\left\|u_{0}\right\|_{2}+\left(\sum_{j=0}^{1} \int_{0}^{t_{n}}\left\|\frac{\partial^{j} g_{2}}{\partial t^{j}}\right\|_{H^{1 / 2}\left(\Gamma_{2}\right)}^{2} \mathrm{~d} s\right)^{1 / 2}\right\}
$$

for $n=1,2, \ldots, N$.

Proof. By Sobolev inequality, we have

$$
\left\|\eta^{n}\right\|_{L^{\infty}} \leqslant C\left(\log \frac{1}{h}\right)^{1 / 2}\left\|\eta^{n}\right\|_{1} .
$$

The desired estimate now follows from Lemma 5.2, Theorem 4.2, (5.4) and the triangle inequality. This completes the rest of the proof.

\section{Acknowledgements}

The authors wish to thank the anonymous referee for his valuable comments and suggestions. The research of the first author has been supported by the Department of Science and Technology, Government of India under BOYSCAST fellowship. The first author also thanks the Institute for Scientific Computations and the Department of Mathematics, Texas A\&M University for providing necessary facilities for carrying out the research work. 


\section{References}

[1] R.A. Adams, Sobolev Spaces, Academic Press, New York, 1975.

[2] S.C. Brenner, L.R. Scott, The Mathematical Theory of Finite Element Methods, Springer, New York, 2002.

[3] Z. Cai, On the finite volume element method, Numer. Math. 58 (1991) 713-735.

[4] Z. Cai, S. McCormick, On the accuracy of the finite volume element method for diffusion equations on composite grids, SIAM J. Numer. Anal. 27 (1990) 636-655.

[5] P. Chatzipantelidis, Finite volume methods for elliptic PDE's: A new approach, Math. Modelling Numer. Anal. 36 (2002) $307-324$.

[6] P. Chatzipantelidis, R.D. Lazarov, V. Thomee, Error estimates for the finite volume element method for parabolic equations in convex polygonal domains, Numer. Methods Partial Differential Equations 20 (2004) 650-674.

[7] S.H. Chou, Q. Li, Error estimates in $L^{2}, H^{1}$ and $L^{\infty}$ in covolume methods for elliptic and parabolic problems: A unified approach, Math. Comp. 69 (2000) 103-120.

[8] R.E. Ewing, R.D. Lazarov, Y. Lin, Finite volume element approximations of nonlocal in time one-dimensional flows in porous media, Computing 64 (2000) 157-182.

[9] R.E. Ewing, R.D. Lazarov, Y. Lin, Finite volume element approximations of nonlocal reactive flows in porous media, Numer. Methods Partial Differential Equations 16 (2000) 285-311.

[10] R.E. Ewing, T. Lin, Y. Lin, On the accuracy of the finite volume element method based on piecewise linear polynomials, SIAM J. Numer. Anal. 39 (2002) 1865-1888.

[11] P. Frolkovic, Flux-based method of characteristics for contaminant transport in flowing groundwater, Comput. Visualization Sci. 5 (2002) $73-83$.

[12] P. Frolkovic, H. De Schepper, Numerical modelling of convection dominated transport coupled with density driven flow in porous media, Adv. Water Resourc. 24 (2001) 63-72.

[13] J. Geiser, Radioactive-retardation-reaction-transport-program for the simulation of radioactive waste disposals, Technical Report ISC-0403-MATH, Institute for Scientific Computation, Texas A\&M University, College Station, TX, 2004.

[14] P. Grisvard, Elliptic Problems in Nonsmooth Domains, Pitman, Massachusetts, 1985.

[15] W. Hackbusch, On first and second order box schemes, Computing 41 (1989) 277-296.

[16] H. Jianguo, X. Shitong, On the finite volume element method for general self-adjoint elliptic problems, SIAM J. Numer. Anal. 35 (1998) $1762-1774$

[17] M. Luskin, R. Rannacher, On the smoothing property of the Galerkin method for parabolic equations, SIAM J. Numer. Anal. 19 (1981) 93-113.

[18] I.D. Mishev, Finite Volume and Finite Volume Element Methods for Non-Symmetric Problems, PhD thesis, Technical Report ISC-96-04MATH, Institute for Scientific Computation, Texas A\&M University, College Station, TX, 1997.

[19] I.D. Mishev, Finite volume methods on Voronoi meshes, Numer. Methods Partial Differential Equations 16 (1998) $193-212$.

[20] V. Thomée, Galerkin Finite Element Methods for Parabolic Problems, Springer, New York, 1997. 